\title{
Electromyographic Grasp Recognition for a Five Fingered Robotic Hand
}

\author{
Nayan M. Kakoty, Mantoo Kaibarta and Shyamanta M. Hazarika \\ School of Engineering, Tezpur University, Tezpur, INDIA
}

\begin{tabular}{ll}
\hline \hline Article Info & ABSTRACT \\
\cline { 2 - 2 } Article history: & $\begin{array}{l}\text { This paper presents classification of grasp types based on surface electromyo- } \\
\text { graphic signals. Classification is through radial basis function kernel support vec- }\end{array}$ \\
Received July, 29, 2012 & tor machine using sum of wavelet decomposition coefficients of the EMG signals. \\
Revised October, 26, 2012 & In a study involving six subjects, we achieved an average recognition rate of $86 \%$. \\
Accepted October 30, 2012 & The electromyographic grasp recognition together with a 8-bit microcontroller has \\
Keyword: & been employed to control a five fingered robotic hand to emulate six grasp types \\
used during 70\% daily living activities.
\end{tabular}

Grasp Types

Electromyogram

sum of wavelet coefficients

Copyright (C) 2012 Insitute of Advanced Engineeering and Science.

\section{Corresponding Author:}

Nayan M. Kakoty

School of Engineering

Tezpur University

Tezpur, INDIA

nkakoty@tezu.ernet.in

\section{INTRODUCTION}

Electromyogram (EMG) signal classification to detect various upper-limb movements have been used for many applications, including hand prosthesis control and human computer interface for last two decades. Hiraiwa et al. has classified five finger movements based on single channel EMG using FFT analysis [1]. Classification of four grasp modes using principal component analysis and Mehalanobis distance function based on four channel EMG signals has been reported by Vuskovic [2]. Nishikawa et al. has reported ten forearm motions discrimination based on two channel EMG using real time learning method [3]. Chan et al. has classified four hand functions based on single channel EMG [4]. Crawford et al. shows the control of four degrees of freedom (DOF) robotic arm based seven channel EMG with 90\% accuracy using linear kernel support vector machine for eight class classification of hand movements [5].

Despite serious research in the field of rehabilitation robotics, not much has been achieved for grasps manipulation by prostheses. Ferguson and Dunlop [6] have reported grasp types classification based on EMG, wherein four types of grasps have been classified with an average recognition rate of $75-80 \%$ using four channel EMG signals. Martelloni et al. [7] have performed the classification of three grasp types based on eight channel EMG with a recognition rate of 84-93\%. Both Fergunsons and Martellonis architectures are subject dependent. More recently, results on grasp type recognition, reported by Castellini et al. [8] is limited to the classification of only three distinct types of grasps using ten surface electrodes with a recognition rate of $90 \%$. Castellini et al. [9] has shown the classification of two grasp types based on seven channel EMG signals with a recognition rate of $97 \%$. Kakoty and Hazarika [10] has reported the classification of six grasp types with an average recognition rate of $97.5 \%$ using two channel EMG.

Almost all literature reports only on the classification of EMG signals and their application for controlling finger movements in robotic hand, not for robotic hand grasping operations [11]. For details in EMG based robotic hand control, see Oskoei et al. [12]. Antflok et.al [13] had successfully rehabilitated 
an amputee using 16 channel EMG signals and controlled a hand prosthetic known as the Smart Hand. Classification of the signals was done using local approximation and lazy learning. The feature used was mean value of preprocessed EMG signals. The experimentation achieved an accuracy rate of $86 \%$ and has been implemented to a robotic hand for performing three grasp types used during daily living activities (dla). Only recently, Cipriani et al. has reported the development of SmartHand capable of performing three grasp types used during dla [14].

The work presented in this paper stems from the desire to create an advanced prosthetic system capable of preshaping and grasping. The focus is on development of an electromyographic control for a five fingered robotic hand: TU Bionic Hand [15]. We concentrated on six grasp types used during $70 \%$ of dla. The classification of six grasp types is based on two channel EMG signals through radial basis function (RBF) kernel support vector machine (SVM). Sum of wavelet decomposition (SWC) coefficients has been used as feature for classification of grasp types based on EMG signals. An average recognition rate of $86 \%$ is achieved. The developed control architecture has been employed to control the five fingered robotic hand emulating the six grasp types.

Rest of the paper is arranged as follows: Section 2. discusses grasp types and EMG signals. The derivation of the feature set is in Section 3. Electromyographic control based on crasp recognition is in section 4. The grasp classification through SVM and recognition results and feed forward control of five fingered robotic hand are discussed in section 4.1. and 4.2. The paper concludes with final comments in section 5 .

\section{GRASP TYPES AND ELECTROMYOGRAM}

\subsection{Grasp Types}

For activities such as lifting, lowering, carrying, pushing and pulling etc., grasp is the type of interface between the subject's hand and the object to be handled. For control of the prosthetic hand, we aim to identify the basic grasp shapes made by the user. An extensive list of grasp types has been reported by M. R. Cutkosky [16]. Based on the wrist orientation, grasp span and strength involved, grasps are categorized into six different types i.e. power, pinch, precision, oblique, hook and palm-up [17]. This classification of grasps for different common operations has been found closer to one in Heumer et al. [18] rather than one given by Fiex et al. [19]. Heumer et al. identified six different grasp types, whereas Fiex et al. has a classification that identifies seventeen grasp types. Six grasp types, as shown in Figure 1 are significant for they are involved in $70 \%$ of dla [20].

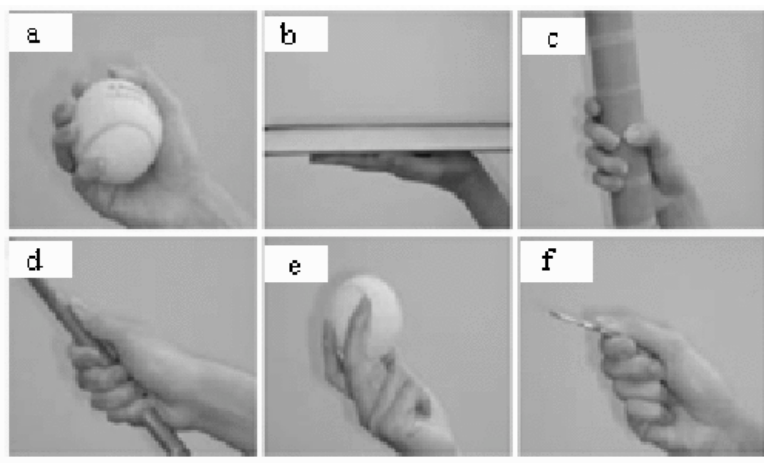

Figure 1. Grasp types: a. Power b. Palm-up c. Hook d. Oblique e. Precision and f. Pinch

\subsection{Electromyogram Acquisition and Integrated Electromyogram}

\subsubsection{Action Potential and Electromyogram Signals}

Muscle fibers are innervated by neurons whose cell bodies are located in spinal cord. The combination of a single motor neuron and all the muscle fibers it innervates is called a motor unit. In response to an action potential from the neuron, a muscle fiber depolarizes as the signal propagates along its surface and 
the fiber contracts. This depolarization followed by repolarization generates an electric field in the vicinity of the muscle fibers. The resulting signal is called the muscle fiber action potential. The combination of the muscle fiber action potentials from all the muscle fibers of a single motor unit is the motor unit action potential (MUAP). The schematic diagram of motor unit action potential is shown in Figure 2.

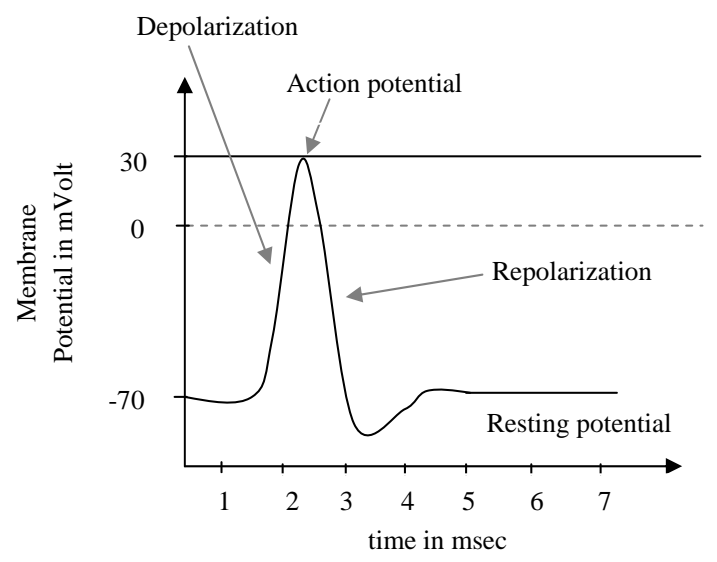

Figure 2. Motor Unit Action Potential showing Depolarization, Repolarization and Resting Potential

The summation of action potentials created by each active motor unit is the electromyogram signal which can be detected by a skin surface electrode. They generally are in the range of $10 \mathrm{~Hz}$ to $10 \mathrm{kHz} ; 10$ $\mu \mathrm{V}$ to $15 \mathrm{mV}$. The type, number and firing rates of motor units involved in grasping operations varies during different grasps [21]. The EMG signals generated during different grasp types epitomize it.

\subsubsection{Electromyogram Acquisition}

Obtaining accurate data from EMG signals depends partially on specific electrode placement and site preparation. One of the most important consideration in site preparation is reducing the amount of impedance between electrode and skins surface. Electrode gel was applied to the site of electrode placement in order to further increase its performance. We acquired EMG signals continuously from the state of the preshaping initiation by the upper limb for a particular grasp type till it takes a closure mode. The aim of doing so is to collect informations about the attempted grasp types from the EMG onset prior to grasp is formed. $\mathrm{Ag} / \mathrm{AgCl}$ buttom type surface electrodes are used. In line with the work by Crawford et al. 2005, the placement of the electrodes on the subjects forearm is tabulated in Table 1 [5]. Two channel EMG signals were recorded for a period of $250 \mathrm{msec}$. This is to meet the real time constraint that the response time of myoelectric control system should be less than $300 \mathrm{msec}$ [22].

Table 1. Placement of EMG Electrodes

\begin{tabular}{|c|c|l|}
\hline Electrode Number & Electrode Leads & Specific Muscle \\
\hline Electrode1 & Lead1 & Extensor Digitorum Muscle \\
& Lead2 & Flexor Digitorum Muscle \\
\hline Electrode2 & Lead1 & Flexor Carpi Ulnaris Muscle \\
& Lead2 & Extensor Carpi Radialis \\
& & Longus Muscle \\
\hline
\end{tabular}

After a hand amputation, much of the forearm remain and can still be used by the amputee. Although our study used healthy subjects, there is evidence that amputees who have lost their hand are able to generate EMG signals from the remanent forearm muscles that are very similar to those generated by healthy subjects $[5,23]$. EMG could thus can be read from these and used as the control source for prosthetic device $[6]$. 


\subsubsection{Integrated Electromyogram}

The raw EMG signals obtained needs to be preprocessed for accurate record and display for further processing. They are passed through high pass, low pass and notch filter using AD Power Lab 4/25 T. The EMG signals obtained after filtration and amplification are called integrated electromyogram (IEMG). Table 2 illustrates the specification setting of the EMG unit during EMG acquisition.

Table 2. EMG Unit specification settings during Signal Acquisition

\begin{tabular}{|l|l|}
\hline Parameter & Value \\
\hline CMRR & $110 \mathrm{~dB}$ \\
\hline Low pass cut off & $2 \mathrm{kHz}$ \\
\hline High pass cut off & $10 \mathrm{~Hz}$ \\
\hline Notch filter cut off & $50 \mathrm{~Hz}$ \\
\hline Amplification range & $+/-5 \mathrm{~V}$ \\
\hline
\end{tabular}

\section{FEATURE SET}

Features are the informations extracted from the signal and can characterize the signal with a smaller set of data. Wavelet transform (WT) is a multiresolution representation that expresses signal variation at different scales. Being highly transitional with sharp peaks and discontinuities, EMG signal analysis with WT is advantageous compared to Fourier transform. SWC can be inferred as the difference between two approximations at subsequent scales [24] and corresponds to the frequency components of the original signal $[25,26]$. During grasping, the number of motor units firing, varies according to the involvement of the forearm extensor and flexor muscles [21]. Further, the firing rate of motor units associated with control and co-ordination of finger movements during grasping operations, varies according to the grasp type [27]. Consequently EMG for each of the grasp types is the composite of different frequency components. We used SWC as the feature for classification of grasp types [28].

\subsection{Derivation of Feature Set}

Let $X(t)$ be the raw EMG signal. The IEMG signal $X_{i}(t)$ is obtained from raw EMG signal by amplification and filtering operations. $X_{i}(t)$ is expressed as:

$$
X_{i}(t)=g \int_{\omega_{1}}^{\omega_{2}} \hat{X}(t) d \omega
$$

where

$\hat{X}(\mathrm{t})=$ EMG signal after $50 \mathrm{~Hz}$ notch filtering

$g=$ gain of the EMG unit

$\omega_{1}, \omega_{2}=$ Low and high cutoff frequency of the band pass filter

DWT decomposes a signal into an approximation signal and detail signal. The detail coefficients $D_{j}$ and the approximation coefficients $A_{j}$ at level j can be obtained by filtering the signal with an L-sample high pass filter g, and an L-sample low pass filter h. Both approximation and detail signals are down sampled by a factor of two [29]. This can be expressed as follows:

$$
\begin{aligned}
& A_{j}[n]=H\left\langle A_{j}-1[n]\right\rangle=\sum_{k=0}^{L-1} h[k] A_{j}-1[2 n-k] \\
& D_{j}[n]=G\left\langle D_{j}-1[n]\right\rangle=\sum_{k=0}^{L-1} g[k] A_{j}-1[2 n-k]
\end{aligned}
$$


where $\mathrm{H}$ and $\mathrm{G}$ represent the convolution/ down sampling operators. Sequences $\mathrm{g}[\mathrm{n}]$ and $\mathrm{h}[\mathrm{n}]$ are associated with wavelet function $\psi(t)$ and the scaling function $\phi(t)$ through inner products:

$$
\begin{aligned}
& g[n]=\langle\psi(t), \sqrt{2} \psi(2 t-n)\rangle \\
& h[n]=\langle\phi(t), \sqrt{2} \phi(2 t-n)\rangle
\end{aligned}
$$

The approximate coefficients contain the most important information of the signal [30] and is therefore used for deriving the feature set. The sum of the Haar wavelet coefficients $\rho$ is computed and input as the feature vector to the classifier. The input feature $\rho$ is given as:

$$
\rho=\sum_{n=1}^{N} A_{j}[n]
$$

where $\mathrm{N}$ is the total number of Haar wavelet coefficients.

\section{ELECTROMYOGRAPHIC CONTROL BASED ON GRASP RECOGNITION}

Figure 3 shows the schematic diagram of EMG based control architecture. The fundamental units are the EMG Unit, Feature Extraction Unit and the Classifier Unit. The EMG unit comprises of the amplifier, band pass and notch filter. The filtered signals were sampled with $5 \mathrm{kHz}$ sampling frequency. The features were extracted from the IEMG signals in the feature extraction unit. The extracted feature is fed to the classifier. Classification is through a RBF Kernel One-vs-All SVM clustering six grasp types.

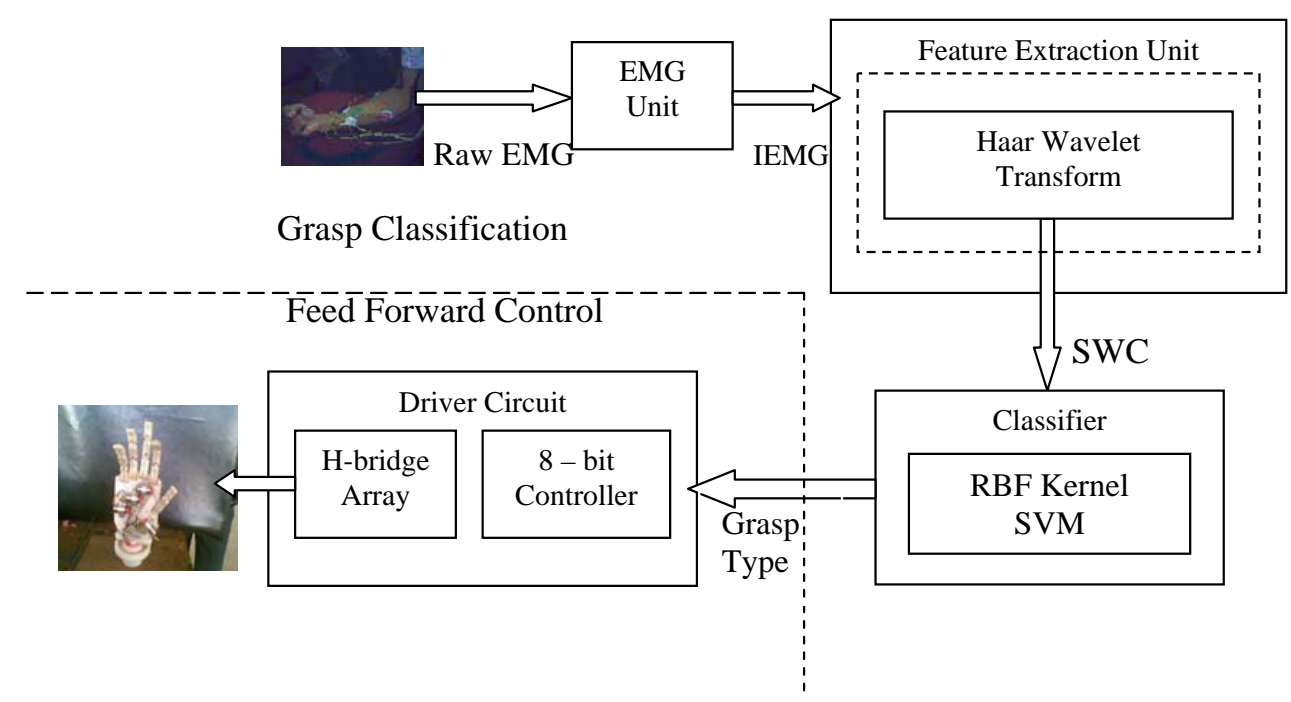

Figure 3. Electromyographic Control Architecture for a Five Fingered Robotic Hand

\subsection{Grasp Classification}

\section{SVM for Classification}

For recognition of grasp types based on the extracted feature, we chose to employ was a RBF kernel SVM. In terms of selecting a kernel function to use with the SVM, there is no method that can determine what kernel function should be used for a particular application. According to [31], the RBF kernel should be the first choice. Another reason to use the RBF kernel is that there are less difficulties with mathematical computations. SVM implicitly map the input data into the feature space where a decision boundary separates the classes that may exist. For the classification of non linear and highly transitional data, the formulation of linear hyperplane is extended to build non linear SVM kernel. Non linear kernel transforms the input 
data into higher dimensional feature space where data can be linearly separated by applying linear SVM formulation [27].

In SVM classifier, the input feature set $\left(\rho_{x}\right.$ and $\left.\rho_{y}\right)$ of two channel IEMG for all grasp types were mapped into high dimensional feature space by $\mathrm{RBF}$ kernel $K$ as:

$$
\left\langle\rho_{x}, \rho_{y}\right\rangle \rightarrow K\left(\rho_{x}, \rho_{y}\right) \quad \text { where } \quad K\left(\rho_{x}, \rho_{y}\right)=e^{\left[\|\left(\rho_{x}-\rho_{y}\right)^{2} \mid / \sigma^{2}\right]}
$$
is given by

with $\sigma$ being the scale factor. The hyperplane separating the feature vectors in higher dimensions

$$
f(x)=b+\sum_{i=1}^{F} w_{i} \cdot Y_{i} \quad \text { satisfying } \quad \min \left[\phi\left(w_{i}\right)\right]=1 / 2\left(w_{i} \cdot w_{i}\right)
$$

where $b=$ bias term, $F=$ total number of input features, $w=$ normal to the feature spaces and

$$
\begin{array}{lllll}
Y_{i}=+1 & \text { if } & w_{i} \rho+b>1 & \text { or } \\
Y_{i}=-1 & \text { if } & w_{i} \rho+b<1 &
\end{array}
$$

\section{Training and Testing}

During training, six subjects performs the grasp types taken under study. All subjects performs each grasps in six trials. The training phase was accomplished with a total of 216 two channel EMG signals. The feature set is extracted from IEMG signals of $480 \mathrm{msec}$. Training for each grasp type takes $560 \mathrm{~ms}$ on a Pentium 2 $\mathrm{GHz}$ processor. In testing, six subjects perform each grasps types eight times randomly. A total of 288 two channel EMG signals were used for testing of the proposed architecture. The resulting hyperplanes of SVM plots lead to six grasp types. Testing for one grasp type takes $520 \mathrm{msec}$ on a Pentium $2 \mathrm{GHz}$ processor.

\section{Recognition Rates}

The classification and misclassification rates of grasp recognition is shown in Figure 4. For power grasp,

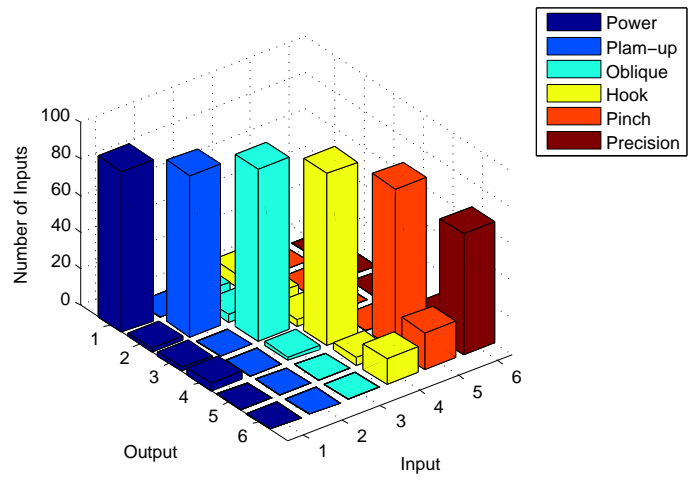

Figure 4. Confusion Matrix for Classification of Six Grasp Types based on SWC

$88 \%$ is correctly classified; whereas $5 \%$ is misclassified as oblique and $7 \%$ as hook. For palm-up grasp, $88 \%$ is classified correctly; whereas $2 \%$ is misclassified as power, $4 \%$ is misclassified as oblique and another $6 \%$ is misclassified as hook. For oblique grasp, $94 \%$ is correctly classified whereas $2 \%$ is misclassified as power, $4 \%$ is misclassified as hook. For hook grasp, $94 \%$ is classified correctly whereas $4 \%$ is misclassified as power and $2 \%$ is misclassified as oblique. For pinch grasp, $88 \%$ is classified correctly whereas $4 \%$ is misclassified as hook and $8 \%$ is misclassified as precision. For precision grasp, $66 \%$ is classified correctly whereas $14 \%$ is misclassified as hook and $20 \%$ is misclassified as pinch. The average recognition rate in classifying the testing data into six grasp types is $86 \%$ and is tabulated in Table 3 . 
Table 3. Recognition Rates

\begin{tabular}{|l|c|}
\hline Grasp Type & Recognition Rate \\
\hline Power & $88 \%$ \\
\hline Palm-up & $88 \%$ \\
\hline Oblique & $94 \%$ \\
\hline Hook & $94 \%$ \\
\hline Pinch & $88 \%$ \\
\hline Precision & $66 \%$ \\
\hline
\end{tabular}

\section{2. $\quad$ Feed Forward Control}

The grasp recognition results from the Classification Unit have been implemented on TU Bionic Hand - a five fingered prosthetic hand designed and developed at Tezpur University, India - shown in Figure 5. For details of the TU Bionic Hand, please refer to our earlier paper [15]. As shown in Figure 5, the motors M1, M3, M5, and M7 are for the flexion of the index finger; middle finger; ring and little finger (in conjunction) and thumb. These are placed on the ventral side; the corresponding complementary motors M2, M4, M6, M8 are for extension of index finger; middle finger; ring and little finger in conjunction and thumb are placed on the dorsal side of the hand. Motor M9 is for inward motion of the palm in order to achieve stable grasp. Motors M10, M11 and M12 are placed mutually perpendicular to each other for achieving 3 DoF at the wrist. Table 4 details the mapping of the identified grasp type into the five fingered robotic hand. After

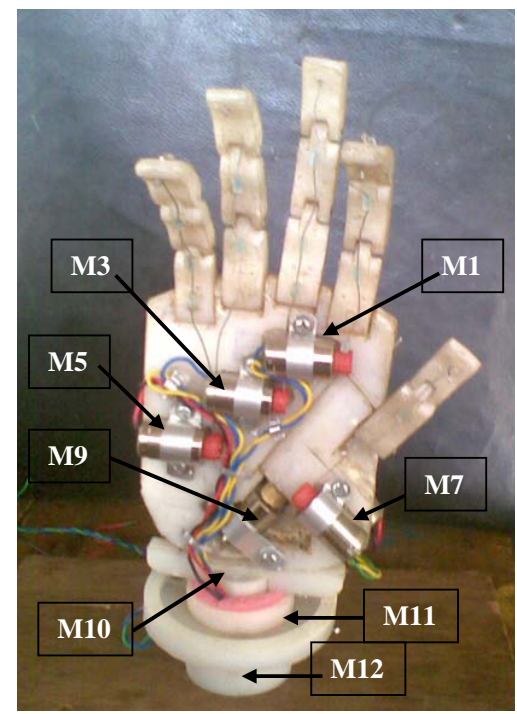

Figure 5. TU Bionic Hand: Ventral View

performing each grasp type, the corresponding complementary motors are actuated in order to have the hand in normal pose. Figure 6 shows the hand performing a precision grasp.

\section{FINAL COMMENTS}

We present the results in discrimination of six grasp types: hook, oblique, palm-up, pinch, power and precision and report $86 \%$ grasp recognition. SWC has been used as the feature for grasp classification. Following an EMG based control, the five fingered robotic hand performs the grasping operations involved during $70 \%$ daily living activities. We are continuing work to improve both the success rate and scope of our controller with a aim to evolve a system wherein a disabled person makes a mental plan of execution of the gesture he naturally feels for a given task and the prosthetic device executes the move! 
Table 4. Mapping of Identified Grasp Types into the TU Bionic Hand

\begin{tabular}{|l|c|c|c|}
\hline $\begin{array}{l}\text { Grasp } \\
\text { Type }\end{array}$ & $\begin{array}{c}\text { Command to } \\
\text { Microcontroller } \\
\text { Port } 0\end{array}$ & $\begin{array}{c}\text { Command from Microcontroller } \\
\text { Port 1, 2 and } 3 \text { to } \\
\text { H-Bridge Circuit }\end{array}$ & Actuated Motors \\
\hline Power & $01 \mathrm{H}$ & Port 1: 11 H, Port 2: 81 H & M1, M3, M5, M7, M9 \\
\hline palm-up & $64 \mathrm{H}$ & $\begin{array}{c}\text { Port 1: } 48 \mathrm{H}, \text { Port 2: } 86 \mathrm{H} \\
\text { Port 3: 01 H }\end{array}$ & M2, M4, M6, M8, M9 \\
\hline Oblique & $02 \mathrm{H}$ & Port 1: 11 H, Port 2: 01 H & M1, M3, M5, M8 \\
\hline Hook & $32 \mathrm{H}$ & Port 1: $11 \mathrm{H}$, Port 2: $41 \mathrm{H}$ & M1, M3, M5, M7, M9 \\
\hline Pinch & $16 \mathrm{H}$ & Port 1: $01 \mathrm{H}$, Port 2: $80 \mathrm{H}$ & M1, M3, M7, M9 \\
\hline Precision & $04 \mathrm{H}$ & Port 1: $80 \mathrm{H}$, Port 2: $11 \mathrm{H}$ & M1, M3, M7 \\
\hline
\end{tabular}

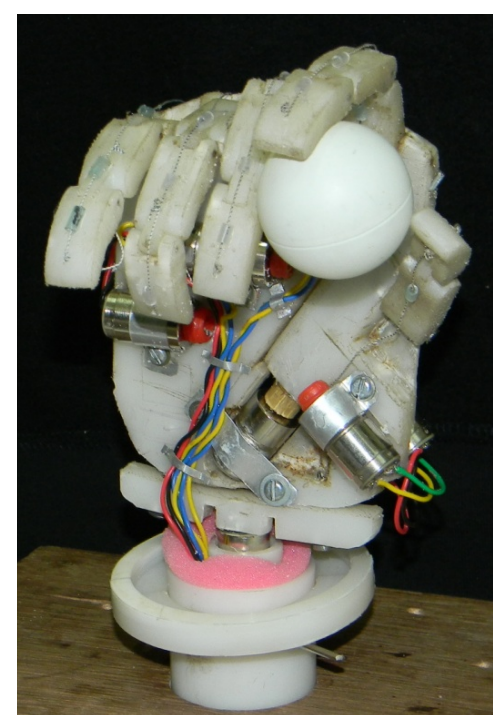

Figure 6. TU Bionic Hand: Performing Precision Grasp

\section{ACKNOWLEDGMENT}

We acknowledge the financial support received from DIT, GoI through its project Design and Development of Cost-effective Bio-signals Controlled Prosthetic Hand under Grant No.1(9)/2008-ME \& TMD.

\section{REFERENCES}

[1] A. Hiraiwa, K. Shimohara, and Y. Tokunaga, "EMG pattern analysis and classification by neural network," in IEEE Intl. Conference on Systems, Man and Cybernatics, USA, 1989, pp. 711-719.

[2] M. I. Vuskovic, A. L. Pozos, and R. Pozos, "Classification of grasp modes based on electromyographic patterns of prehensing motions," IEEE trans. of Systems, Mam and Cyb., vol. 1, no. 1, pp. 89-95, 1995.

[3] D. Nishikawa, W. Yu, H. Yokoi, and Y. Kakazu, "EMG prosthetic hand controller using real-time learning method," in IEEE Intl. Conf. on Systems Man and Cybernatics, USA, 1999.

[4] F. H. Y. Chan, Y.-S. Yang, F. K. Lam, Y.-T. Zhang, and P. A. Parker, "Fuzzy EMG classification for prosthesis control," IEEE Trans. on Rehabilitation Engineering, vol. 8, no. 3, pp. 305-311, 2000.

[5] B. Crawford, K. Miller, P. Shenoy, and R. Rao, "Real-time classification of electromyographic signals for robotic control," Dept. of Computer Science, University of Washington, Tech. Rep. 2005-03-05, 2005.

[6] S. Ferguson and G. R. Dunlop, "Grasp recognition from myoelectric signals," in Proceedings of Australian Conference on Robotics and Automation, Auckland, 2002, pp. 82-84.

[7] C. Martelloni, J. Carpaneto, and S. Micera, "Classification of upper arm EMG signals during objectspecific grasp," in In 30th Annual International IEEE/ EMBS Conference, Canada, 2008, pp. 2061-5064.

[8] C. Castellini, E. Fiorilla, and G. Sandini, "Multi-subject/ DLA analysis of surface EMG control of mechanical hands," in poster at GNB I, First Italian Bioengineering Congress, Italy, Pisa, Italy, 2008. 
[9] C. Castellini and S. Patrick, "Surface emg in advanced hand prosthetics," Biological cybernetics, vol. 100, no. 1, pp. 35-47, 2009.

[10] N. M. Kakoty and S. M. Hazarika, "Recognition of grasp types through PCs of DWT based EMG features," in Intl. Conf. on Rehab. Robotics, Zurich, Switzerland, 2011, pp. 478-482.

[11] P. Shenoy, K. J. Miller, B. Crawford, and R. N. Rao, "Online electromyographic control of a robotic prosthesis," IEEE Trans. Biomed. Eng., vol. 55, pp. 1128-1135, 2008.

[12] M. Oskoei and H. Hu, "Myoelectric control systemsa survey," J. of Biomed. Signal Proc. Control, vol. 2, pp. 275-294, 2007.

[13] C. Antfolk, C. Cipriani, M. Controzzi, M. C. Carrozza, G. Lundborg, B. Rosen, and F. Sebelius., "Using emg for real-time prediction of joint angles to control a prosthetic hand equipped with a sensory feedback system," Journal of Medical and Biological Engineering, vol. 30, no. 6, pp. 399-406, 2010.

[14] C. Cipriani, M. Controzzi, and M. C. Carrozza, "The smarthand transradial prosthesis," Journal of NeuroEngineering and Rehabilitation, vol. 8, no. 29, pp. 1-13, 2011.

[15] N. M. Kakoty and S. M. Hazarika, "Biomimetic design and development of a prosthetic hand: Prototype 1.0," in 15th National Conference on Machine and Mechanisms, IIT Madras, INDIA, 2011, pp. 499-506.

[16] M. R. Cutkosky, "On grasp choice, grasp models and the design of hands for manufacturing tasks," Robotics and Auto., vol. 5, no. 3, pp. 269-278, 1989.

[17] C. MacKenzie and T. Iberall, TheGrasping Hand. Amesterdam: North-Holland, 1994.

[18] G. Heumer, H. B. Amar, and B. Jung, "Grasp recognition for un-calibrated data gloves: A machine learning approach," J. of Medical Systems, vol. 17, no. 2, pp. 121-142, 2008.

[19] T. Fiex, R. Pawlik, H. B. Schmiedmayer, J. Romero, and D. Kragic, "A comprehensive grasp taxonomy," in Workshop on Understanding the Human Hand for Advancing Robotic Manipulation; Workshop at Robotics: Science and Systems Conference, Austria, June, 2009.

[20] F. Vecchi, S. Micera, M. C. Carrozza, A. M. Sabatini, and P. Dario, "A sensorized glove for applications in biomechatronics and motor control," in 6th IFESS Conference, San Francisco, CA, 2008, pp. 346-353.

[21] S. L. Kilbreath, R. B. Gorman, J. Raymond, and S. C. Gandevia, "Distribution of the forces produced by motor unit activity in the human flexon digitorium profundus," J. of Physiology, vol. 543, no. 1, pp. 289-296, 2002.

[22] A. Phinyomark, C. Limsakul, and P. Phukpattaranont, "A novel feature extraction for robust emg pattern recognition," Journal of Computing, vol. 1, pp. 71-80, 2009.

[23] L. Eriksson, F. Sebelius, and C. Balkenius, "Neural control of a virtual prosthesis," in International Conference on Artificial Neural Network, London, 1998.

[24] J. Ellis, N. E. Mavromatos, D. V. Napopoulos, and A. S. Sakharov, "Quantum-gravity analysis of gamma-ray burst using wavelets," J. of Astronomy and Astrophysics, vol. 402, no. 2, pp. 409-424, 2003.

[25] S. Karasawa, "Discrete wavelet transform based multiple teplate-matching for speech recognition," in Pattent Application No: 2006-357183, Japan, 2007.

[26] S. Karasawa and H. Sakuraba, "Use of haar wavelet transform based multiple teplate matching for analyses of speech voice," in Proc. of Euro-American Conf. on Tel. and Info. System, Portugal, 2007.

[27] B. E. Boser, I. M. Guyon, and V. N. Vapnik, "A training algorithm for optimal margin classifiers," in 5th Annual ACM Workshop on COLT, Pittsburgh, 1992.

[28] N. M. Kakoty and S. M. Hazarika, "Classification of grasp types through wavelet decomposition of EMG signals," in IEEE Conf. on Biomedical Engineering and Informatics, China, 2009.

[29] I. R. Carreno and M. Vuskovic, "Wavelet transform momonts for feature extraction from temporal signals," Informatics in Control, Automation and Robotics, vol. 2, no. 3, pp. 235-242, 2007.

[30] S. Li, J. Liao, and J. T. Kwok, "Wavelet-based feature extraction for microarray data classification," in IEEE International Conference on Neural Network, Canada, 2006, pp. 5028-5033.

[31] C. C. C. C.-W. Hsu and C. J. Lin, "A practical guide to support vector classification," 2009, available at http://www.csie.ntu.edu.tw/ cjlin/libsvm. 


\section{BIOGRAPHY OF AUTHORS}
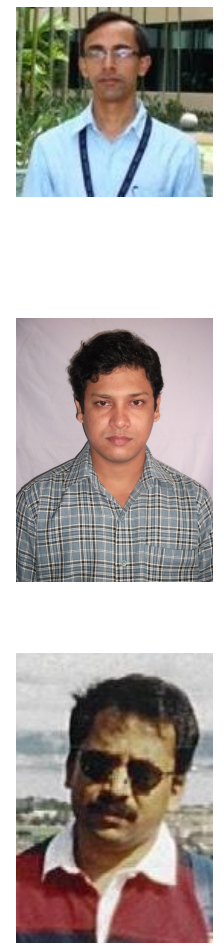

Nayan M. Kakoty is a research scholar in the Cognitive and Rehabilitation Robotics Laboratory, School of Engineering, Tezpur University, INDIA. His research focus is biosignals controlled extrem upper limb prosthetic hand. He has completed BE in Electrical Engineering and M. Tech. in BioElectronics. He is affiliated with IEEE and Association for Machines \& Mechanisms as member.

Mantoo Kaibarta is a Project Engineer in the Cognitive and Rehabilitation Robotics Laboratory, School of Engineering, Tezpur University, INDIA. He has completed M. Sc. in Instrumentation and M. Tech. in BioElectronics.

Shyamanta M. Hazarika is a Professor of Computer Science and Engineering at Tezpur University. He completed his BE from Assam Engineering College, Guwhati, M.Tech in Robotics from IIT Kanpur, and PhD from University of Leeds, England. He has been a Full Professor for Cognitive Systems and NeuroInformatics at the Cognitive Systems Group, University of Bremen, Germany, for Winter 2009-2010. His work has focused primarily in knowledge representation and reasoning and rehabilitation robotics with an aim towards development of intelligent assistive systems. 